
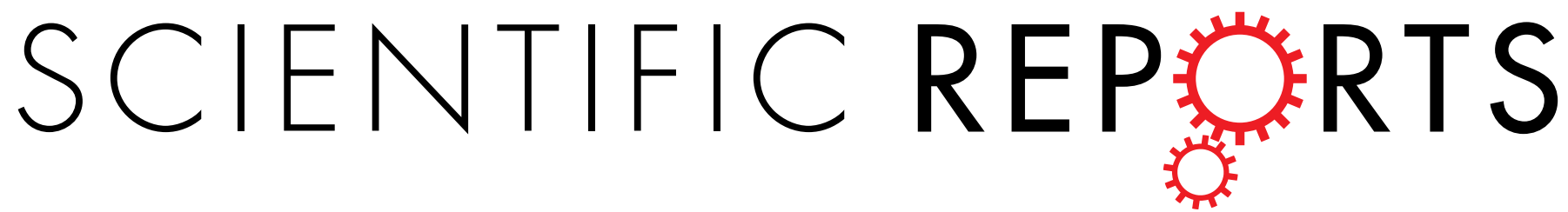

\title{
OPEN Additive enhancement of wound healing in diabetic mice by low level light and topical CoO10
}

Received: 09 April 2015

Accepted: 15 December 2015

Published: 02 February 2016
Zhigang Mao ${ }^{1,2}$, Jeffrey H. Wu ${ }^{2}$, Tingting Dong² \& Mei X. Wu ${ }^{2}$

Diabetes, a highly prevalent disease that affects $9.3 \%$ of Americans, often leads to severe complications and slow wound healing. Preclinical studies have suggested that low level light therapy (LLLT) can accelerate wound healing in diabetic subjects, but significant improvements must be made to overcome the absence of persuasive evidence for its clinical use. We demonstrate here that LLLT can be combined with topical Coenzyme $\mathrm{O} 10$ (CoO10) to heal wounds in diabetic mice significantly faster than LLLT alone, CoO10 alone, or controls. LLLT followed by topical CoO10 enhanced wound healing by 68 103\% in diabetic mice in the first week and more than $24 \%$ in the second week compared with untreated controls. All wounds were fully healed in two weeks following the dual treatment, in contrast to only $50 \%$ wounds or a fewer being fully healed for single or sham treatment. The accelerated healing was corroborated by at least $50 \%$ higher hydroxyproline levels, and tripling cell proliferation rates in LLLT and $\mathrm{CoO} 10$ treated wounds over controls. The beneficial effects on wound healing were probably attributed to additive enhancement of ATP production by LLLT and CoO10 treatment. The combination of LLLT and topical CoO10 is safe and convenient, and merits further clinical study.

There are an estimated 371 million cases of diabetes worldwide, putting patients at serious risk for wound healing complications that reduce both quality and length of life. In particular, foot ulcers hamper $15 \%$ of all diabetics, accounting for the majority of non-traumatic lower limb amputations in the United States ${ }^{1}$. These complex wounds result from a combination of diminished immune function, peripheral neuropathy, arterial occlusion, and miscellaneous factors such as limited joint mobility and improper footwear ${ }^{1-3}$. Small sores are readily healed in healthy individuals but frequently develop into deep ulcers, leading to infections of underlying tissues and bones that sometimes necessitate amputation in diabetic patients. Treatment of these chronic wounds in diabetic patients mainly relies on conventional wound bed preparation, mechanical and surgical debridement, and antibiotic therapy to battle infections $s^{4,5}$. However, these managements have extremely limited efficacy, leading to a high 5 -year mortality rate following amputation ${ }^{6}$.

Considering the toll on patients and $\$ 11$ billion cost to American health care payers ${ }^{7}$, there is a substantial demand for better approaches to diabetic wound management. The potential of low-level laser therapy (LLLT), a non-invasive treatment that some studies have shown to successfully accelerate wound healing in specific circumstances, is particularly attractive ${ }^{8}$. LLLT does not cause a noticeable temperature change in subjects, yet seems to stimulate tissue repair and relieve pain ${ }^{8-10}$. While their efficacy in clinical settings has long been disputed ${ }^{11}$, an increasing number of studies have contended that LLLT can increase fibroblast proliferation, collagen production, angiogenesis, granulation formation, and other positive effects ${ }^{12}$. As for diabetes specifically, a study proved that $633 \mathrm{~nm}$ laser at $10 \mathrm{~J} / \mathrm{cm}^{2}$ accelerated wound healing in diabetic rats by $\sim 38 \%{ }^{1}$. Systematic reviews have been hindered by lack of adequate sample sizes and consistent methodologies in clinical studies, but demonstrate plausibility and safety of using LLLT to treat diabetic wounds².

Therapeutic mechanisms of LLL are not well understood to date. Considering that thermal increases are absent following LLLT, photobiomodulation may be the major contributor of the reported acceleration in tissue repair $^{8,12,13}$. For example, one event suspected to contribute significantly to wound healing is the absorption of far red or near infrared light $(600-1100 \mathrm{~nm})$ by the redox active metal centers of cytochrome c oxidase, also known as complex IV of the electron transport chain at mitochondria ${ }^{12}$. Such absorption is thought to increase

${ }^{1}$ ShangHai 9th People Hospital, Shanghai Jiao Tong University School of Medicine, ShangHai, China. ${ }^{2}$ Wellman Center for Photomedicine, Massachusetts General Hospital, Department of Dermatology, Harvard Medical School, Boston, MA 02114. Correspondence and requests for materials should be addressed to M.X.W. (email: mwu5@mgh. harvard.edu) 


\begin{tabular}{|c|c|c|c|c|c|c|}
\hline \multicolumn{2}{|c|}{ Name of the 4 groups } & \multirow{2}{*}{$\begin{array}{c}\text { Treatments } \\
\text { sham light+vehicle }\end{array}$} & \multirow{2}{*}{$\begin{array}{c}\text { Wounds } \\
12\end{array}$} & \multirow{3}{*}{\begin{tabular}{|c} 
Animals \\
12
\end{tabular}} & \multirow{3}{*}{$\begin{array}{c}\begin{array}{c}\text { Sacrificed } \\
\text { on day } 7\end{array} \\
6\end{array}$} & \multirow{3}{*}{$\begin{array}{c}\begin{array}{c}\text { Sacrificed } \\
\text { on day } 16\end{array} \\
6\end{array}$} \\
\hline Thoracic & Control & & & & & \\
\hline Lumbar & LLLT & LLLT+vehicle & 12 & & & \\
\hline Thoracic & CoQ10 & sham light $+\mathrm{CoQ} 10$ & 12 & \multirow{2}{*}{12} & \multirow{2}{*}{6} & \multirow{2}{*}{6} \\
\hline Lumbar & LLLT+CoQ10 & LLLT+CoQ10 & 12 & & & \\
\hline
\end{tabular}

Table 1. Experiment design.

mitochondria respiration activity and ATP synthesis ${ }^{8,13}$. Likewise, ubiquinone CoQ10 can enhance mitochondrial activity by virtue of being a cofactor in the complexes I-III in the mitochondrial electron transport chain ${ }^{14,15}$. Besides, CoQ10 acts as a potent anti-oxidant and displays anti-ulcer potential ${ }^{15-18}$. Because LLL and CoQ10 target different complexes in the mitochondrial electron transport chain, these two modalities may additively accelerate diabetic wound healing.

With this in mind, we tested and found that LLLT followed by topical CoQ10 resulted in diabetic wound healing significantly faster than individual treatment or sham-treated controls. The dual treatment is simple, safe, and holds great promise for future management of diabetic wounds in the clinics.

\section{Materials and Methods}

Animals. The experimental subjects were 5-week-old male Swiss Webster mice (Charles river lab, USA) weighing 20-30 g. The mice were maintained in a temperature-controlled room under a $12 \mathrm{~h}$ light $/ 12 \mathrm{~h}$ dark cycle and provided ad libitum access to food and water. All animal experiments were performed according to the National Institutes of Health guidelines for the Care and Use of Laboratory Animals and approved by the Subcommittee on Research Animal Care of the Massachusetts General Hospital.

Animal model and study design. To induce diabetes in mice, streptozotocin ( $120 \mathrm{mg} / \mathrm{kg}$, Sigma) in sodium citrate buffer ( $\mathrm{pH} 4.0$ ) was injected intraperitoneally after fast for $4 \sim 6 \mathrm{hr}$. Measurement with Blood Glucose Meter (Germaine Laboratories) confirmed diabetic state, which was conducted for three consecutive days after one week of streptozotocin injection and verified one day before surgical wounds. Mice exhibiting glucose levels over $200 \mathrm{mg} / \mathrm{dl}$ for all four tests were considered to be diabetic and enrolled to the study. On day 30 after streptozotocin injection, the animals were shaved on the dorsal skin after anesthetized by intraperitoneal administration of ketamine $(90 \mathrm{mg} / \mathrm{kg}) /$ xylazine $(10 \mathrm{mg} / \mathrm{kg})$. Two full-thickness, $1 \mathrm{~cm}^{2}$ square defects were made on the thoracic and lumbar regions of each animal, respectively, one to receive a treatment and another to serve as a corresponding control such that the treatment and corresponding control could be compared on the same subject as listed in the Table 1 . The 24 mice were randomly selected for different treatments with 12 wounds in each group. Half of the mice in each group were sacrificed for skin sampling on day 7 and the remaining mice were sacrificed on day 16. Control wounds experienced sham light and then topical vehicle (soybean oil) application, while the rest of the wounds received LLLT + topical vehicle (LLLT), sham light + topical CoQ10 (CoQ10) or LLLT followed by topical CoQ10 (LLLT+CoQ10). LLLT was applied to the wound areas every two days starting in 24 hours after wound formation using an $830 \mathrm{~nm}$ infrared light emitting diode (LED) from Omnilux new-U, USA. The average irradiance of the light was $14 \mathrm{~mW} / \mathrm{cm}^{2}$ for a total exposure time of 5 minutes or an energy density of $4.2 \mathrm{~J} / \mathrm{cm}^{2}$ on the surface of the wound as measured by Handheld Laser Power \& Energy Meter (Ophir Nova II). During the illumination, the mouse was covered by aluminum sheet with an opening area of $1 \mathrm{~cm}^{2}$ to expose the wound, at which LLL was directed vertically at a distance of $6 \mathrm{~cm}$ above. The sham light was administered similarly with a small soft white LED lightbulb ( $3 \mathrm{~W}$ A15) from General Electric (GE) such that no heat was generated on the wound. The skin temperature during 5 minutes of illumination increased $\leq 0.1^{\circ} \mathrm{C}$ with either light source. After illumination, CoQ10 was topically applied to the wound so that there was not any direct interaction between LLL and CoQ10. An over-the-counter CoQ10 supplement $(30 \mathrm{mg} / \mathrm{ml}$, Bio Quinone) was diluted by 5 times in soybean oil and $50 \mu \mathrm{l}$ of the CoQ10 solution was gently wiped onto the surface of the wound immediately post-LLLT. The wound was covered 20 minutes later by a bandage to protect from mouse bites or scratching.

Wound-healing measurement. Photographs of each full-thickness skin defect, next to a ruler for calibration, were taken using an Olympus digital camera. Wound area in square centimeters was analyzed using Image J software and repeated measurements were accurate within $2 \%$. The first images were obtained on the day of injury (day 0). Subsequent images were captured on the third, seventh, and thirteenth days post-injury. The wound healing areas were measured by percentages of a healed area relative to an original wound area using the following formula: The healed area percentage $=\left(\right.$ original wound area - unepithelialized area)/original wound area $^{19}$, where healing referred to a combination of wound constriction and epithelization, not including crust formation and granulation. The measurement was conducted in a manner blinded to the study groups.

Cell proliferation assay. Cell proliferation in situ was analyzed with 5-ethynyl-2' -deoxyuridine (EdU) assays from Invitrogen. EdU is a thymidine analogue and incorporated into the DNA of dividing cells. Mice were injected intraperitoneally with $100 \mu \mathrm{g}$ of EdU in PBS on day 6 after wounds and then the skin specimens were collected one day later ${ }^{20}$. Two specimens were excised from each wound, one from the center of the wound bed and the other from uninjured adjacent skin served as reference such that a total of 4 specimens were collected from each mouse. The skin specimens were formalin-fixed, embedded in paraffin, and cut into $5 \mu \mathrm{m}$ thick sections. For EdU staining, paraffin in the tissue sections was removed and the tissue was stained with the Click-iT ${ }^{\circledR}$ EdU Alexa Fluor $^{\circledR} 488$ Imaging Kit (Invitrogen, USA), counterstained with DNA dye DAPI (4',6-diamidino-2-phenylindole) 
provided in the kit per the manufacturer's instruction. The stained sections were imaged by confocal microscopy (Olympus FV1000, Olympus, Japan) and analyzed by Image J software. Percentages of EdU-positive cells relative to a total number of cells were obtained by manually counting the number of EdU-positive green cells and DAPI-positive blue cells in 100 microscopic fields randomly selected in each sample in a sample-blind manner.

Hydroxyproline levels. Hydroxyproline levels were used to evaluate collagen content in the extracts of healing skin tissues. Specimens were excised and weighed from the wound bed and uninjured adjacent skin as above. The skin sample of $5 \mathrm{mg}$ was homogenized in $100 \mu \mathrm{l}$ of water before transfer to a pressure-tight vial with PTFE-lined cap and addition of $100 \mu \mathrm{l}$ concentrated hydrochloric acid $(\mathrm{HCl}, \sim 12 \mathrm{M})$. It was then capped tightly and hydrolyzed at $120^{\circ} \mathrm{C}$ for 3 hours. The resultant supernatant $(20 \mu \mathrm{l})$ was transferred to a 96 -well plate and placed in a $60^{\circ} \mathrm{C}$ oven to dry. Finally, the samples were measured using a Hydroxyproline Assay Kit (Sigma, USA). Hydroxyproline was quantified by $560 \mathrm{~nm}$ absorbance with a microplate reader (Molecular Devices, Sunnyvale, CA, USA).

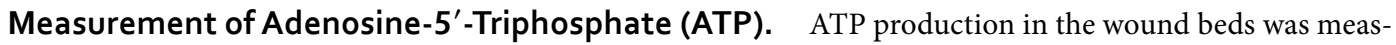
ured $1 \mathrm{hr}$ after the first indicated treatments using an ATP assay kit (Promega, Madison, WI, USA), in separate groups of mice. Briefly, the tissues from the wound beds were carefully isolated and homogenized in $1 \%$ trichloroacetic acid (sigma, USA). The resultant homogenates were added in duplicate into 96-well luminescence plate containing $100 \mu \mathrm{l}$ of assay reagent. The luminescence signal was measured with a microplate reader (Molecular Devices, Sunnyvale, CA, USA). Relative ATP levels were normalized to the total protein concentration measured by a Bio-Rad protein assay kit.

Statistical Analysis. The data were expressed as mean \pm standard deviation (SD). Statistical analysis was performed by one-way ANOVA and then Tukey's Multiple Comparisons tests among multiple groups using Graph Pad Prism 5.0 (Graph Pad Software, La Jolla, CA, USA). Percentage of completely healed wounds was analyzed by two-sample $t$-test (StatPac, Pepin, WI, USA). A p value $<0.05$ was considered statistically significant.

\section{Results}

LLLT and CoQ10 additively accelerate wound healing. To examine wound-healing responses to LLLT, CoQ10, or both, two full-thickness wounds were made in the dorsal skin of each mouse, one for treatment and the other for the corresponding control. In sham-treated controls, the healed area was $19 \%$ on day $3,30 \%$ on day 7 , and $78 \%$ on day 13 (Fig. 1A-C). In comparison, the dual treatment of LLL and topical CoQ10 significantly accelerated healing to $32 \%$ on day $3(\mathrm{p}<0.05), 61 \%$ on day $7(\mathrm{p}<0.001)$, and $97 \%$ on day $13(\mathrm{p}<0.001)$. In other words, LLLT followed with topical CoQ10 resulted in $68 \sim 103 \%$ improvement in wound healing during the first week and more than $24 \%$ in the following week. The LLLT plus CoQ10 group outperformed the only LLLT and only CoQ10 groups as well (Fig. 1A-C). While mice treated with LLL healed slower than LLLT+CoQ10 (Fig. 1A-C), they did heal faster than controls in agreement with prior studies showing beneficial effects of LLLT on the wounds of diabetic subjects ${ }^{2}$. For LLLT alone, the healed area was $26 \%$ on day $3,53 \%$ on day 7 ( $\left.p<0.001\right)$, and $91 \%$ on day $13(\mathrm{p}<0.05)$ as compared to sham-treated controls (Fig. 1A-C). On the other hand, the CoQ10 group did not demonstrate statistical significances in wound healing compared to controls. The difference in the percentages of wound healing was significant between LLLT +CoQ10 and only CoQ10 groups at all experimental days tested, which held the truth between LLLT + CoQ10 and only LLLT group on day 3 and day $13(\mathrm{p}<0.05)$, but not on day 7 (Fig. 1A-C). Representative photos following different treatments are shown in Fig. 2, where each row of the figures features the progression of wound healing in a single subject over time, and areas outlined by white dashed lines indicate un-epithelialized areas. Significant reduction in wound area could be seen by the naked eye on day 7 following LLLT plus CoQ10 treatment, as compared to single modality or sham-treated control. Notably, complete re-epithelialization was found in the mice treated with LLL plus CoQ10 by day 13, as shown in the lower right corner of the figure. In accordance with this, about $50 \%$ of wounds showed complete healing on day 13 after treatment with LLL followed by topical CoQ10 and all of the wounds were fully healed by day 15 (Fig. 1D). In contrast, none of sham-treated controls or wounds treated with LLLT or CoQ10 alone demonstrated complete wound healing by day 13, and only 2, 1, or 3 out of 6 healed in the controls or wounds treated with CoQ10 alone or LLL alone by day 15, respectively (Fig. 1D). Complete healing of the wounds receiving LLLT in combination with CoQ10 was highly significant on both days 13 and 15 compared with controls or wounds treated with LLLT or CoQ10 alone $(\mathrm{p}<0.001)$, whereas no difference was found in the wounds treated with LLL alone, CoQ10 alone vs. sham treatment.

Significant increases in hydroxyproline levels by LLLT and CoQ10. Hydroxyproline is a non-proteinogenic amino acid largely restricted to collagen, so its measurement can be used as an indicator of collagen content. The mean hydroxyproline content in the control group was $1.36 \pm 0.15 \mu \mathrm{g} / \mathrm{mg}$ wet tissue on day 7 and increased to $1.58 \pm 0.24 \mu \mathrm{g} / \mathrm{mg}$ on day 16 . In accordance to additive effects of LLLT and CoQ10 on wound healing, the levels of hydroxyproline were elevated to $2.13 \pm 0.24 \mu \mathrm{g} / \mathrm{mg}$ from $1.36 \pm 0.15 \mu \mathrm{g} / \mathrm{mg}$ on day 7 and to $2.48 \pm 0.22 \mu \mathrm{g} / \mathrm{mg}$ from $1.58 \pm 0.24 \mu \mathrm{g} / \mathrm{mg}$ on day 16 in the LLLT+CoQ10 group, which were more than $50 \%$ increases over corresponding control groups $(\mathrm{p}<0.05)$. A high level of hydroxyproline or collagen indicates active healing since collagen increases the strength of the wound and is crucial for many processes at early stages of wound healing. Hydroxyproline values were higher in the LLLT group than in the sham-treated control, but no significant difference could be established between LLLT $(1.81 \pm 0.14 \mu \mathrm{g} / \mathrm{mg}$ on day 7 and $2.08 \pm 0.23 \mu \mathrm{g} / \mathrm{mg}$ on day 16) or CoQ10 $(1.40 \pm 0.13 \mu \mathrm{g} / \mathrm{mg}$ on day 7 and $1.65 \pm 0.14 \mu \mathrm{g} / \mathrm{mg}$ on day 16$)$ and sham-treated controls (Fig. 3). 
A

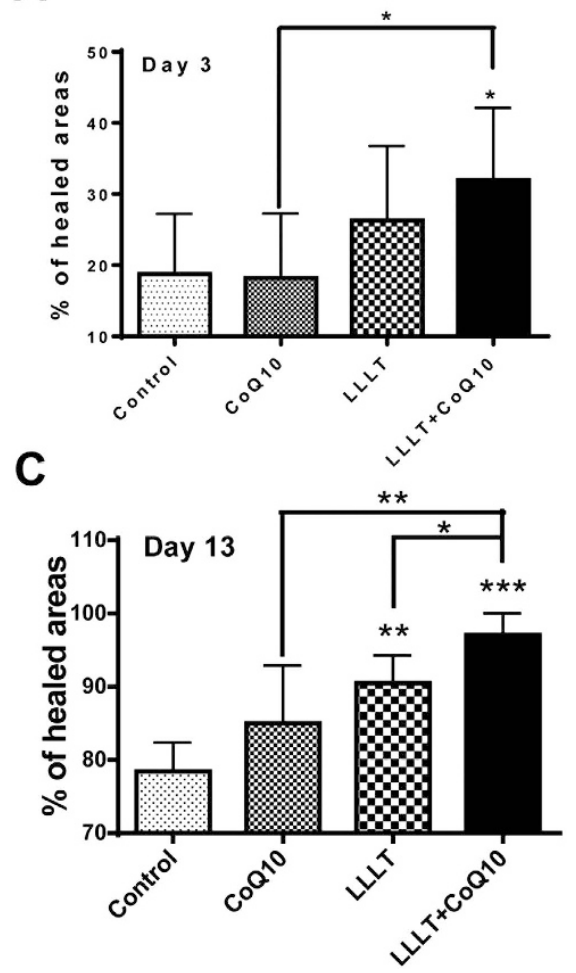

B
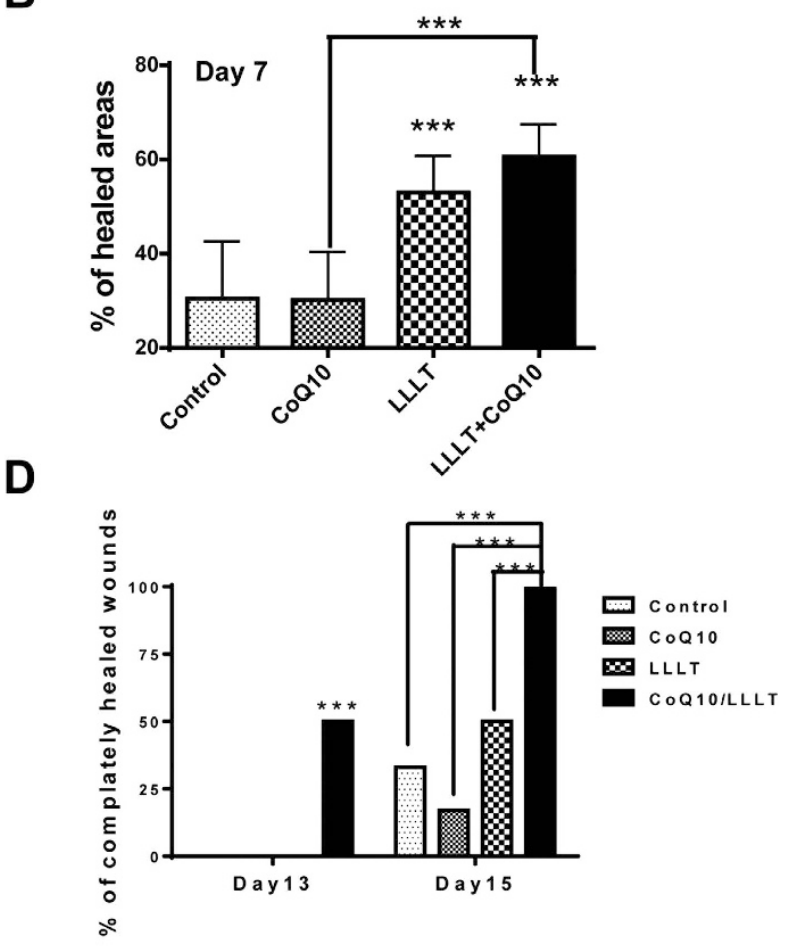

Figure 1. Additive effects of LLLT and CoQ10 on wound healing in diabetic mice. Cutaneous full-thickness wounds were generated in diabetic mice and treated $24 \mathrm{hr}$ later with sham light plus topical vehicle of CoQ10 (control), sham light + topical CoQ10 (CoQ10), LLLT+ vehicle (LLLT), or LLLT followed by topical CoQ10 $(\mathrm{LLLT}+\mathrm{CoQ} 10)$. Wound areas were measured with Image J of photos taken at indicated days post-injury (A-C). The level of wound healing was defined as percentages of re-epithelialized areas over original wound areas as detailed in Materials and Methods. (D) Percentages of wounds with complete healing in each group. Complete wound healing as defined by full epithelialization was tracked in 13 and 15 days. Note: all skin wounds were completely healed in day 15 in LLLT + CoQ10 group only. ${ }^{*} \mathrm{p}<0.05,{ }^{* *} \mathrm{p}<0.01$ and ${ }^{* * *} \mathrm{p}<0.001$ between LLLT+CoQ10 group and all other three groups or between indicated groups. $\mathrm{n}=12$ in each group for data collected before or on day 7 or $n=6$ for data collected after day 7 .

Augmentation of cell proliferation by LLLT and CoQ10. Proliferating cells can be labeled by a thymidine analog EdU and it is simple and robust ${ }^{21}$, allowing consistent results when processing a large number of mouse skin sections. We found that the rate of cell proliferation for controls was $8 \%$ on day 7 , on average, indicating a rather low level of cell proliferation in the absence of any treatment. LLLT and CoQ10 robustly enhanced cell proliferation rate to $24 \%(\mathrm{p}<0.001)$, which represents a $300 \%$ increase over controls, while there were no significant differences in the CoQ10 or LLLT alone group compared with controls (Fig. 4 A,B). Thus, comparison between quantities of EdU positive cells in wounds revealed greatly increased cell proliferation following LLLT and CoQ10 treatment compared to single modality treatment or controls (Fig. 4A). Moreover, consistent with additive or synergic improvement of mitochondrial function by LLLT and CoQ10 treatment, ATP production at the wound bed was increased by two times $1 \mathrm{hr}$ after LLLT but it was three times by a combination of LLLT and CoQ10 compared to the control group (Fig. 4C). In contrast, CoQ10 alone didn't display any significant effect on ATP production (Fig. 4C). Apparently, the level of ATP production at the wound beds was positively and proportionally correlated with the wound healing, similar to previous investigation ${ }^{22}$.

\section{Discussion}

There remain many unmet clinical obstacles to treat diabetic wounds, problems magnified by the consistently rising prevalence of type- 2 diabetes. The lack of pharmacologic treatment options creates urgent need for innovative treatment modalities. Poor angiogenesis perturbs oxygen supply in the wound tissues, so targeting ischemia and hypoxia associated with diabetic wounds has led to some success ${ }^{23}$. For example, vascular grafts bypassing arterial occlusions have in many cases promoted healing for ischemic diabetic wounds ${ }^{24}$. Meanwhile, clinicians use hyperbaric oxygen therapy (HBOT) to provide high levels of oxygen to the wound ${ }^{25}$. Despite promising comparative observational studies, HBOT has failed to demonstrate statistical significance in randomized controlled trials ${ }^{26,27}$.

Alternatively, early studies have shown that LLLT significantly accelerates wound healing in diabetic rats ${ }^{28}$. LLLT offers a non-invasive and easily performed modality to treat diabetic ulcerations, either alone or as a complement procedure. Its mechanism has been elucidated in part by the ability of LLL to enhance the activity of the electron transport chain and ATP production at mitochondria ${ }^{9,12,13}$. We recently subjected non-diabetic mice to 
A

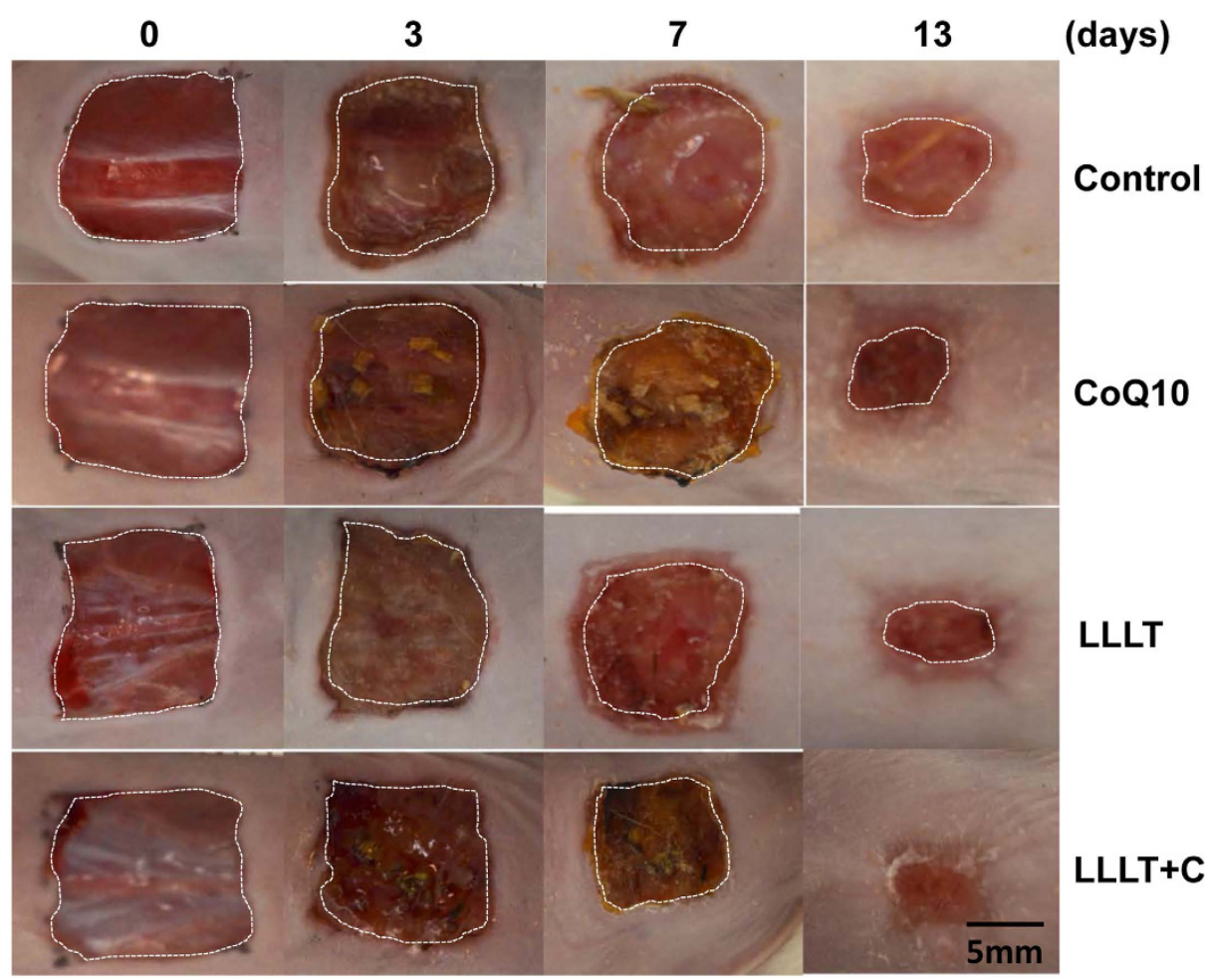

Figure 2. Representative photos of each treatment. Wounds were generated and photos were taken as in Fig. 1. Each row depicts the same wound as it progresses for 13 days. Shown are representative photos from indicated treatments as above. Bar, $5 \mathrm{~mm}$.

A

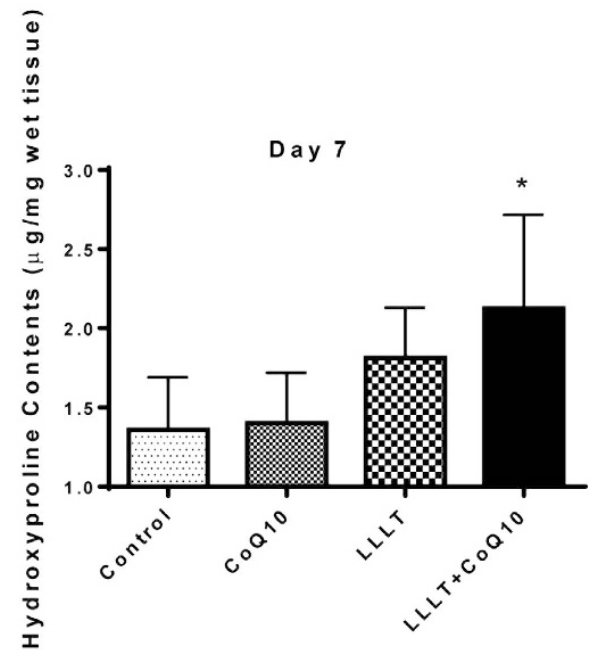

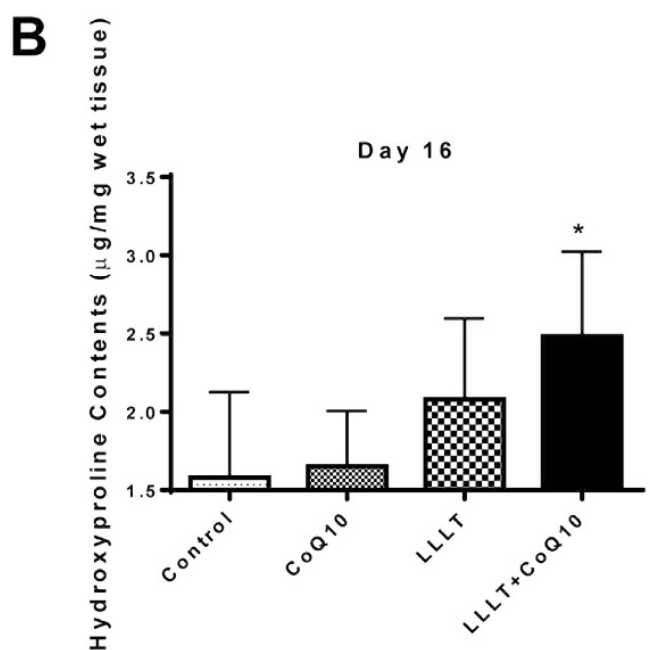

Figure 3. Increased hydroxyproline contents in the wound tissue after treatment with LLLT plus CoQ10. Hydroxyproline contents were measured as $\mu \mathrm{g} / \mathrm{mg}$ wet tissue in 7 (A) or 16 (B) days post-injury. ${ }^{\star} \mathrm{p}<0.05$ between LLLT+CoQ10 group and all other three groups. $n=6$ in each group.

cerebral injury followed by LLLT, corroborating that LLLT at $830 \mathrm{~nm}$ enhanced ATP synthesis as well as reduced production of reactive oxygen species (ROS), inflammation, and neuron apoptosis ${ }^{22}$. The beneficial effect of LLLT sufficiently prevented secondary brain injury and significantly diminished tissue loss following traumatic brain injury, particularly in mice with sub-optimal mitochondrial activity ${ }^{22}$. Our investigations further demonstrated that LLL augmented ATP generation, and suppressed ROS formation or apoptosis in the injured brain subjected to hypoxia ${ }^{29}$. The effect of LLLT was significantly bolstered by combining LLL with metabolic substrates like pyruvate or lactate both in vivo and in vitro as these metabolic substrates can enhance functions of mitochondria even under hypoxic conditions ${ }^{29}$. 
A

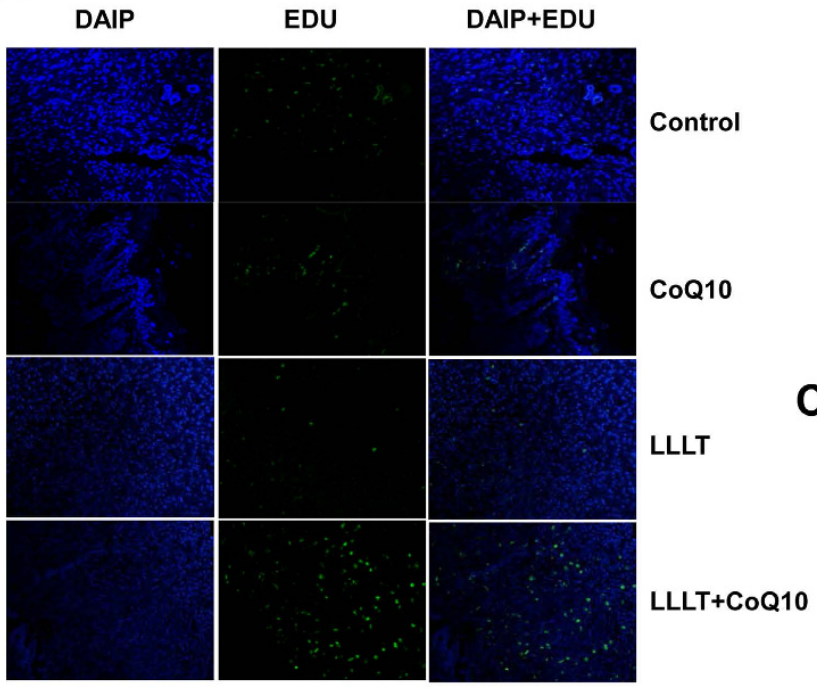

B
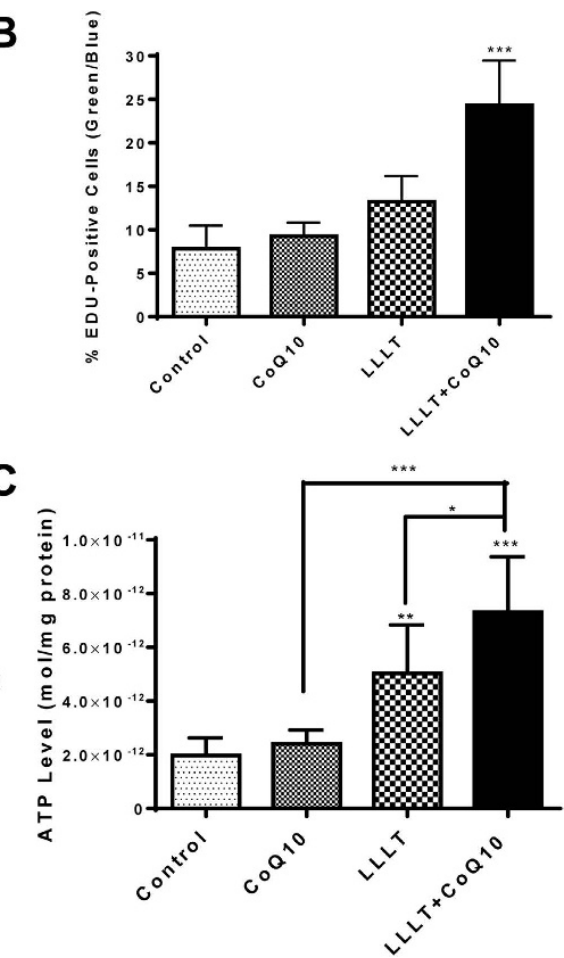

Figure 4. Active cell proliferation in wound tissues following the dual treatment. Cell proliferation was evaluated 7 days after wounds by the EdU assay. (A) Confocal microscopy of EdU-stained tissue sections. Green fluorescence represents EdU-positive cells and DAPI stained all cells. Representative results of 36 sections in each group are shown. Magnification, $\times 20$. (B) EdU-fluorescence intensity. Percentages of EDU-positive cells over a total number of cells are determined by counting individual cells in 100 microscopic fields selected randomly. ${ }^{* *} \mathrm{p}<0.001$ between LLLT + CoQ10 group and all other three groups $(\mathrm{n}=100)$. (C) ATP levels were measured in duplicate $1 \mathrm{hr}$ after the first indicated treatments. ${ }^{*} \mathrm{p}<0.05,{ }^{* *} \mathrm{p}<0.01$ and ${ }^{* * *} \mathrm{p}<0.001$ between LLLT + CoQ10 group and all other three groups or between indicated groups. $n=8$ in each group.

Growing evidence points toward a broader connection between mitochondria and diabetes, despite the fact that only an estimated $0.1-9 \%$ of diabetic cases involve direct contributions from mitochondrial DNA mutations ${ }^{30}$. Such metabolic dysfunction in diabetics is primarily ascribed to inadequate function of mitochondria that increases ROS production and disturbs cellular signaling involved in wound healing, slowing the healing process $^{30-32}$. Thus we employed LLL and CoQ10, also known as ubiquinone, to substantially improve mitochondrial functions in diabetic wounds. Ubiquinone is both a component in the electron transport chain and, when reduced to ubiquinol, an antioxidant. Oral CoQ10 administration is commonly used to address global mitochondrial dysfunction in diabetics and to improve glycemic control and blood pressure over months of treatment ${ }^{33}$. CoQ10 was also administered intraperitoneally, along with $980 \mathrm{~nm}$ LLL treatment to mitigate neuropathic pain in rat daily for two consecutive weeks, but no significant difference was found in the dual treatment relative to single treatment ${ }^{34}$. Likewise, we found no immediate effects on wound healing after administering $1 \mathrm{ml}$ CoQ10 orally every other day for a total of two weeks either alone or in conjunction with LLLT in our initial study (data not shown). Thus we directly applied CoQ10 topically onto wound sites, in hopes that sufficient doses of the quinone could improve mitochondrial function. Indeed, ATP level was elevated additively by LLLT plus CoQ10 as compared to LLLT alone (Fig. 4C), indicating that the dual treatment can better improve mitochondrial function, leading to faster wound healing. Likewise, we previously observed that ATP production at the initial phase of traumatic brain injury was a determinant factor in the control of subsequent wound healing ${ }^{22}$. The level of ATP measured in the early phase of injury was proportionally and inversely correlated with brain tissue loss with a coefficient factor of $0.9^{22}$. Improved mitochondrial function at the early phase of the wound, manifested by increasing ATP production, may sufficiently prevent cell death while greatly augmenting cell proliferation, leading to accelerated healing. Consistent with this, in our study four groups of mice receiving CoQ10 and LLLT, just CoQ10 or LLLT, or sham treatment controls, whether measured by wound area reduction, collagen production, or cell proliferation, all correlate with ATP production at the early phase of the wound, with the combined CoQ10 and LLLT group demonstrating the highest level of ATP production and fastest wound healing among all treatments tested. Our data clearly demonstrate that LLLT followed by topical CoQ10 has a great potential to be alternative treatment of wounds for diabetic patients either alone or as a complement modality. It merits immediate clinical studies because of its simplicity and super safety. 


\section{References}

1. Al-Watban, F., Zhang, X. \& Andres, L. Low-level laser therapy enhances wound healing in diabetic rats: a comparison of different lasers. Photomed. Laser Surg. 25, 72-77 (2007).

2. Beckmann, H., Meyer-Hamme, G. \& Schröder, S. Low level laser therapy for the treatment of diabetic foot ulcers: a critical survey. Evidence-based Complement. Altern. Med. 626127, doi: 10.1155/2014/626127 (2014).

3. Singh, N., Armstrong, D. \& Lipsky, A. Preventing foot ulcers in patients with diabetes. JAMA 293, 217-228 (2005).

4. Schultz, S. et al. Wound bed preparation: A systematic approach to wound management. Wound Repair Regen. 11, Suppl 1, S1-28. (2003).

5. Richard, J., Sotto, A. \& Lavigne, J. New insights in diabetic foot infection. World J. Diabetes 2, 24-32 (2011).

6. Moulik, P., Mtonga, R. \& Gill, G. Amputation and Mortality in New-Onset. Pathophysiology/Complications 26, 491-494 (2003).

7. Driver, V. R., Fabbi, M., Lavery, L. A. \& Gibbons, G. The costs of diabetic foot: the economic case for the limb salvage team. J. Vasc. Surg. 52, 17S-22S (2010).

8. Eells, J. T. et al. Mitochondrial signal transduction in accelerated wound and retinal healing by near-infrared light therapy. Mitochondrion 4, 559-567 (2004).

9. Dungel, P. et al. Low level light therapy by LED of different wavelength induces angiogenesis and improves ischemic wound healing. Lasers Surg. Med. 46, 773-780 (2014).

10. Kim, W.-S. \& Calderhead, R. G. Is light-emitting diode phototherapy (LED-LLLT) really effective? Laser Ther. 20, 205-215 (2011).

11. Posten, W. et al. Low-level laser therapy for wound healing: mechanism and efficacy. Dermatol. Surg. 31, 334-340 (2005).

12. Chaves, A. \& Piancastelli, A. C. C. Effects of low-power light therapy on wound healing: LASER x LED. An. Bras. Dermatol. 89, 616-623 (2014)

13. Silveira, P. et al. Evaluation of mitochondrial respiratory chain activity in muscle healing by low-level laser therapy. J. Photochem. Photobiol. B Biol. 95, 89-92 (2009).

14. Crane, F. L. Biochemical functions of coenzyme Q10. J. Am. Coll. Nutr. 20, 591-598. (2001).

15. Ernster, L. \& Dallner, G. Biochemical, physiological and medical aspects of ubiquinone function. Biochim. Biophys. Acta 1271, 195-204. (1995).

16. Kohli, Y. et al. Effect of hypoxia on acetic acid ulcer of the stomach in rats with or without coenzyme Q10. Jpn. J. Exp. Med. 51, 105-108. (1981).

17. Bentinger, M. et al. The antioxidant role of coenzyme Q. Mitochondrion. 7 Suppl, S41-S50. (2007).

18. Choi, B. S. et al. Effect of coenzyme Q10 on cutaneous healing in skin-incised mice. Arch. Pharm. Res. 32, 907-913 (2009).

19. Watanabe, S. et al. Protective effect of inhalation of hydrogen gas on radiation-induced dermatitis and skin injury in rats. J. Radiat. Res. 55, 1107-1113. (2014).

20. Salic, A. \& Mitchison, T. J. A chemical method for fast and sensitive detection of DNA synthesis in vivo. Proc. Natl. Acad. Sci. USA 105, 2415-2420. (2008).

21. Bordiuk, O. L., Smith, K., Morin, P. J. \& Semenov, M. V. Cell proliferation and neurogenesis in adult mouse brain. PLoS. One. 9, e111453. (2014).

22. Zhang, Q., Zhou, C., Hamblin, M. R. \& Wu, M. X. Low-level laser therapy effectively prevents secondary brain injury induced by immediate early responsive gene X-1 deficiency. J. Cereb. Blood Flow Metab. 34, 1391-401 (2014).

23. Flegg, J., Byrne, H. M., Flegg, M. B. \& Sean McElwain, D. L. Wound healing angiogenesis: The clinical implications of a simple mathematical model. J. Theor. Biol. 300, 309-316 (2012).

24. LoGerfo, F. W. et al. Trends in the care of the diabetic foot. Expanded role of arterial reconstruction. Arch. Surg. 127, 617-620 (1992).

25. Faglia, E. et al. Adjunctive systemic hyperbaric oxygen therapy in treatment of severe prevalently ischemic diabetic foot ulcer. A randomized study. Diabetes Care. 19, 1338-1343 (1996).

26. O'Reilly, D. et al. Hyperbaric oxygen therapy for diabetic ulcers: systematic review and meta-analysis. Int. J. Technol. Assess. Health Care 29, 269-81 (2013).

27. O'Reilly, D. et al. A prospective, double-blind, randomized, controlled clinical trial comparing standard wound care with adjunctive hyperbaric oxygen therapy (HBOT) to standard wound care only for the treatment of chronic, non-healing ulcers of the lower limb in patients wi. Trials 12, 69 (2011).

28. Dancakova, L. et al. Low-level laser therapy with $810 \mathrm{~nm}$ wavelength improves skin wound healing in rats with streptozotocininduced diabetes. Photomed. Laser Surg. 32, 198-204. (2014).

29. Dong, Tingting, Zhang, Qi, Hamblin, Michael, R. \& Wu, M. X. Low-level light in combination with metabolic modulators for effective therapy of injured brain. J. Cereb. Blood Flow Metab. (2015). Available at: http://www.ncbi.nlm.nih.gov/pubmed/25966949 (Accessed: $13^{\text {th }}$ May 2015)

30. Lamson, D. W. \& Plaza, S. M. Mitochondrial factors in the pathogenesis of diabetes: A hypothesis for treatment. Altern. Med. Rev. 7, 94-111 (2002).

31. Senel, O., Cetinkale, O., Ozbay, G., Ahçioğlu, F. \& Bulan, R. Oxygen free radicals impair wound healing in ischemic rat skin. Ann. Plast. Surg. 39, 516-523 (1997).

32. Panchatcharam, M., Miriyala, S., Gayathri, V. S. \& Suguna, L. Curcumin improves wound healing by modulating collagen and decreasing reactive oxygen species. Mol. Cell. Biochem. 290, 87-96 (2006).

33. Hodgson, J. M., Watts, G. F., Playford, D. a, Burke, V. \& Croft, K. D. Coenzyme Q10 improves blood pressure and glycaemic control: a controlled trial in subjects with type 2 diabetes. Eur. J. Clin. Nutr. 56, 1137-1142 (2002).

34. Jameie, S. B. et al. Combined therapeutic effects of low power laser (980nm) and CoQ10 on Neuropathic Pain in adult male rat. Med. J. Islam Repub. Iran 28, 58. (2014).

\section{Acknowledgements}

The authors would like to thank all the members in the Photopathology Core at Wellman Center for experimental assistance with histopathology and microscopy and members in Dr. Hamblin's and Dr. Wu's groups at Wellman Center for Photomedicine for stimulating discussions. This work is supported in part by FA9550-11-1-0331 and FA9550-13-1-0068, Department of Defense/Air Force Office of Scientific Research Military Photomedicine Program, W81XWH-13-2-0067, Department of Defense, CDMRP/BAA, and Wellman Center found to MXW.

\section{Author Contributions}

Z.G.M. designed and performed the research and analyzed data. J.H.W. analyzed the data and wrote the manuscript and T.D. participated in data analysis and manuscript writing. M.X.W. designed and supervised the research and wrote the manuscript.

\section{Additional Information}

Competing financial interests: The authors declare no competing financial interests. 
How to cite this article: Mao, Z. et al. Additive enhancement of wound healing in diabetic mice by low level light and topical CoQ10. Sci. Rep. 6, 20084; doi: 10.1038/srep20084 (2016).

(c) (i) This work is licensed under a Creative Commons Attribution 4.0 International License. The images or other third party material in this article are included in the article's Creative Commons license, unless indicated otherwise in the credit line; if the material is not included under the Creative Commons license, users will need to obtain permission from the license holder to reproduce the material. To view a copy of this license, visit http://creativecommons.org/licenses/by/4.0/ 